PROCEEDINGS OF THE

AMERICAN MATHEMATICAL SOCIETY

Volume 137, Number 8, August 2009, Pages 2557-2565

S 0002-9939(09)09855-4

Article electronically published on March 12, 2009

\title{
THE AVERAGE BEHAVIOR OF FOURIER COEFFICIENTS OF CUSP FORMS OVER SPARSE SEQUENCES
}

\author{
HUIXUE LAO AND AYYADURAI SANKARANARAYANAN
}

(Communicated by Wen-Ching Winnie Li)

\begin{abstract}
Let $\lambda(n)$ be the $n$th normalized Fourier coefficient of a holomorphic Hecke eigenform $f(z) \in S_{k}(\Gamma)$. In this paper we are interested in the average behavior of $\lambda^{2}(n)$ over sparse sequences. By using the properties of symmetric power $L$-functions and their Rankin-Selberg $L$-functions, we are able to establish that for any $\varepsilon>0$,
\end{abstract}

$$
\sum_{n \leq x} \lambda^{2}\left(n^{j}\right)=c_{j-1} x+O\left(x^{1-\frac{2}{(j+1)^{2}+2}+\varepsilon}\right),
$$

where $j=2,3,4$.

\section{INTRODUCTION AND MAIN RESULTS}

Let $S_{k}(\Gamma)$ be the space of holomorphic cusp forms of even integral weight $k$ for the full modular group $\Gamma=\mathrm{SL}(2, \mathbb{Z})$. Suppose that $f(z)$ is an eigenfunction of all Hecke operators belonging to $S_{k}(\Gamma)$. Then the Hecke eigenform $f(z)$ has the following Fourier expansion at the cusp $\infty$ :

$$
f(z)=\sum_{n=1}^{\infty} a(n) e^{2 \pi i n z}
$$

where we normalize $f(z)$ such that $a(1)=1$. Instead of $a(n)$, one often considers the normalized Fourier coefficient

$$
\lambda(n)=\frac{a(n)}{n^{\frac{k-1}{2}}} .
$$

Then $\lambda(n)$ is real and satisfies the multiplicative property

$$
\lambda(m) \lambda(n)=\sum_{d \mid(m, n)} \lambda\left(\frac{m n}{d^{2}}\right),
$$

where $m \geq 1$ and $n \geq 1$ are any integers. The Fourier coefficients of cusp forms are interesting objects. In 1974, P. Deligne [2] proved the Ramanujan-Petersson

Received by the editors October 17, 2008.

2000 Mathematics Subject Classification. Primary 11F30, 11F11, 11F66.

Key words and phrases. Fourier coefficients of cusp forms, symmetric power $L$-function, Rankin-Selberg $L$-function.

This work is supported by the National Natural Science Foundation of China (Grant No. 10701048).

(C)2009 American Mathematical Society Reverts to public domain 28 years from publication 
conjecture

$$
|\lambda(n)| \leq d(n),
$$

where $d(n)$ is the divisor function. For the sum of normalized Fourier coefficients over natural numbers, Rankin [14] proved that

$$
S(x)=\sum_{n \leq x} \lambda(n) \ll x^{\frac{1}{3}}(\log x)^{-\delta},
$$

where $0<\delta<0.06$.

In 2001, Ivić [5] studied the sum of normalized Fourier coefficients over squares, i.e.

$$
S_{2}(x)=\sum_{n \leq x} \lambda\left(n^{2}\right) .
$$

By (1.1), the Rankin-Selberg method, and the zero-free region of the Riemann zeta function, he successfully gave a nontrivial estimate

$$
S_{2}(x) \ll_{f} x \exp \left(-A(\log x)^{\frac{3}{5}}(\log \log x)^{-\frac{1}{5}}\right),
$$

where $A$ is a suitable positive constant.

Later Fomenko [3] mentioned that

$$
S_{2}(x) \ll_{f} x^{\frac{1}{2}}(\log x)^{3} .
$$

Recently Sankaranarayanan [16] showed that

$$
S_{2}(x) \ll x^{\frac{3}{4}}(\log x)^{\frac{19}{2}} \log \log x
$$

holds uniformly for any holomorphic cusp form of even integral weight $k$ for the full modular group satisfying $k \ll x^{\frac{1}{3}}(\log x)^{\frac{22}{3}}$. In the same paper, Sankaranarayanan mentioned that it is an open problem to give a nontrivial estimate for the sum of Fourier coefficients over cubes, i.e.

$$
S_{3}(x)=\sum_{n \leq x} \lambda\left(n^{3}\right) .
$$

Recently by using the properties of symmetric power $L$-functions, Lü [12 proved that for any $\varepsilon>0$,

$$
S_{3}(x)=\sum_{n \leq x} \lambda\left(n^{3}\right) \ll_{f, \varepsilon} x^{\frac{3}{4}+\varepsilon}, \quad S_{4}(x)=\sum_{n \leq x} \lambda\left(n^{4}\right) \ll_{f, \varepsilon} x^{\frac{7}{9}+\varepsilon} .
$$

On the other hand, Rankin [13] and Selberg [17] studied the average behavior of $\lambda^{2}(n)$ over natural numbers and showed that

$$
\sum_{n \leq x} \lambda^{2}(n)=c x+O_{f}\left(x^{\frac{3}{5}}\right)
$$

Therefore a natural problem is what is the average behavior of $\lambda^{2}(n)$ over sparse sequences. In this paper we are interested in this problem, namely to study the asymptotic formula of the sum

$$
\sum_{n \leq x} \lambda^{2}\left(n^{j}\right)
$$

where $j=2,3,4$. 
By using the properties of symmetric power $L$-functions and their Rankin-Selberg $L$-functions, which have been established in [4], 8], [9], [10], 11], and [18, we are able to establish the following results.

Theorem 1.1. Let $f(z) \in S_{k}(\Gamma)$ be a Hecke eigenform of even integral weight $k$ for the full modular group, and let $\lambda(n)$ denote its nth normalized Fourier coefficients. Then for any $\varepsilon>0$, we have

$$
\sum_{n \leq x} \lambda^{2}\left(n^{2}\right)=c_{1} x+O_{f, \varepsilon}\left(x^{\frac{9}{11}+\varepsilon}\right) .
$$

Theorem 1.2. Let $f(z) \in S_{k}(\Gamma)$ be a Hecke eigenform of even integral weight $k$ for the full modular group, and let $\lambda(n)$ denote its nth normalized Fourier coefficients. Then for any $\varepsilon>0$, we have

$$
\sum_{n \leq x} \lambda^{2}\left(n^{3}\right)=c_{2} x+O_{f, \varepsilon}\left(x^{\frac{8}{9}+\varepsilon}\right) .
$$

Theorem 1.3. Let $f(z) \in S_{k}(\Gamma)$ be a Hecke eigenform of even integral weight $k$ for the full modular group, and let $\lambda(n)$ denote its nth normalized Fourier coefficients. Then for any $\varepsilon>0$, we have

$$
\sum_{n \leq x} \lambda^{2}\left(n^{4}\right)=c_{3} x+O_{f, \varepsilon}\left(x^{\frac{25}{27}+\varepsilon}\right) .
$$

\section{Some Lemmas}

Lemma 2.1. Let $f(z) \in S_{k}(\Gamma)$ be a Hecke eigenform of even integral weight $k$ for the full modular group, and let $\lambda(n)$ denote its nth normalized Fourier coefficients. For $j=2,3,4$, we introduce

$$
L_{j}(s)=\sum_{n=1}^{\infty} \frac{\lambda^{2}\left(n^{j}\right)}{n^{s}}
$$

for $\operatorname{Re}(s)>1$. Let $L\left(\operatorname{sym}^{j} f, s\right)$ be the jth symmetric power L-function associated to $f$, and let $L\left(\mathrm{sym}^{j} f \times \mathrm{sym}^{j} f, s\right)$ be the Rankin-Selberg L-function of $\mathrm{sym}^{j} f$ and $\operatorname{sym}^{j} f$.

Then we have that for $\operatorname{Re}(s)>1$,

$$
L_{j}(s)=L\left(\operatorname{sym}^{j} f \times \operatorname{sym}^{j} f, s\right) U_{j}(s),
$$

where $U_{j}(s)$ converges uniformly and absolutely in the half-plane $\operatorname{Re}(s) \geq 1 / 2+\varepsilon$ for any $\varepsilon>0$.

Proof. According to Deligne [2, for any prime number $p$ there are $\alpha(p)$ and $\beta(p)$ such that

$$
\lambda(p)=\alpha(p)+\beta(p) \text { and }|\alpha(p)|=\alpha(p) \beta(p)=1 .
$$

Then it is easy to show that for $j \geq 1$,

$$
\lambda\left(p^{j}\right)=\frac{\alpha(p)^{j+1}-\beta(p)^{j+1}}{\alpha(p)-\beta(p)}=\sum_{m=0}^{j} \alpha(p)^{j-m} \beta(p)^{m} .
$$

In fact for any integer $j \geq 2$, from the theory of Hecke operators we have the following recursive relation:

$$
\lambda\left(p^{j}\right)=\lambda\left(p^{j-1}\right) \lambda(p)-\lambda\left(p^{j-2}\right) .
$$


By induction, we have

$\lambda\left(p^{j}\right)=\frac{\alpha(p)^{j}-\beta(p)^{j}}{\alpha(p)-\beta(p)} \times(\alpha(p)+\beta(p))-\frac{\alpha(p)^{j-1}-\beta(p)^{j-1}}{\alpha(p)-\beta(p)}=\frac{\alpha(p)^{j+1}-\beta(p)^{j+1}}{\alpha(p)-\beta(p)}$.

Therefore we have

$$
\lambda^{2}\left(p^{j}\right)=\left(\sum_{m=0}^{j} \alpha(p)^{j-m} \beta(p)^{m}\right)^{2} .
$$

The $j$ th symmetric power $L$-function attached to $f \in S_{k}(\Gamma)$ is defined as

$$
L\left(\operatorname{sym}^{j} f, s\right):=\prod_{p} \prod_{m=0}^{j}\left(1-\alpha(p)^{j-m} \beta(p)^{m} p^{-s}\right)^{-1}
$$

for $\operatorname{Re}(s)>1$. The Rankin-Selberg $L$-function associated to $\operatorname{sym}^{j} f$ and $\operatorname{sym}^{j} f$ is defined as

$$
L\left(\operatorname{sym}^{j} f \times \operatorname{sym}^{j} f, s\right):=\prod_{p} \prod_{m=0}^{j} \prod_{u=0}^{j}\left(1-\alpha(p)^{j-m} \beta(p)^{m} \alpha(p)^{j-u} \beta(p)^{u} p^{-s}\right)^{-1}
$$

for $\operatorname{Re}(s)>1$. The product over primes also gives a Dirichlet series representation for $L\left(\operatorname{sym}^{j} f \times \operatorname{sym}^{j} f, s\right)$ : for $\operatorname{Re}(s)>1$,

$$
L\left(\operatorname{sym}^{j} f \times \operatorname{sym}^{j} f, s\right)=\sum_{n=1}^{\infty} \frac{\lambda_{\operatorname{sym}^{j} f \times \operatorname{sym}^{j} f}(n)}{n^{s}},
$$

where $\lambda_{\operatorname{sym}^{j} f \times \operatorname{sym}^{j} f}(n)$ is a multiplicative function. From (2.3), we have

$$
\left|\lambda_{\mathrm{sym}^{j} f \times \operatorname{sym}^{j} f}(n)\right| \leq d_{(j+1)^{2}}(n),
$$

where $d_{k}(n)$ is the $n$th coefficient of the Dirichlet series $\zeta^{k}(s)$. Then we have that for $\operatorname{Re}(s)>1$,

$L\left(\operatorname{sym}^{j} f \times \operatorname{sym}^{j} f, s\right)=\prod_{p}\left(1+\frac{\lambda_{\mathrm{sym}^{j} f \times \mathrm{sym}^{j} f}(p)}{p^{s}}+\cdots+\frac{\lambda_{\mathrm{sym}^{j} f \times \mathrm{sym}^{j} f}\left(p^{k}\right)}{p^{k s}}+\cdots\right)$.

By (2.6) and (2.8), we have

$$
\begin{aligned}
\lambda_{\mathrm{sym}^{j} f \times \operatorname{sym}^{j} f}(p) & =\sum_{m=0}^{j} \sum_{u=0}^{j} \alpha(p)^{j-m} \beta(p)^{m} \alpha(p)^{j-u} \beta(p)^{u} \\
& =\left(\sum_{m=0}^{j} \alpha(p)^{j-m} \beta(p)^{m}\right)^{2} .
\end{aligned}
$$

From (2.4) and (2.9), we find that

$$
\lambda^{2}\left(p^{j}\right)=\lambda_{\mathrm{sym}^{j} f \times \operatorname{sym}^{j} f}(p) .
$$


From (1.2), we learn that

$$
L_{j}(s)=\sum_{n=1}^{\infty} \frac{\lambda^{2}\left(n^{j}\right)}{n^{s}}
$$

is absolutely convergent in the half-plane $\operatorname{Re}(s)>1$. On noting that $\lambda^{2}\left(n^{j}\right)$ is a multiplicative function, we have that for $\operatorname{Re}(s)>1$,

$$
L_{j}(s)=\sum_{n=1}^{\infty} \frac{\lambda^{2}\left(n^{j}\right)}{n^{s}}=\prod_{p}\left(1+\frac{\lambda^{2}\left(p^{j}\right)}{p^{s}}+\frac{\lambda^{2}\left(p^{2 j}\right)}{p^{2 s}}+\cdots+\frac{\lambda^{2}\left(p^{k j}\right)}{p^{k s}}+\cdots\right) .
$$

Therefore from $(2.8),(2.10)$ and $(2.12)$, we have that for $\operatorname{Re}(s)>1$,

$$
\begin{aligned}
L_{j}(s)= & L\left(\operatorname{sym}^{j} f \times \operatorname{sym}^{j} f, s\right) \\
& \times \prod_{p}\left(1+\frac{\lambda^{2}\left(p^{2 j}\right)-\lambda_{\operatorname{sym}^{j} f \times \operatorname{sym}^{j} f}\left(p^{2}\right)}{p^{2 s}}+\cdots\right) \\
=: & L\left(\operatorname{sym}^{j} f \times \operatorname{sym}^{j} f, s\right) U_{j}(s) .
\end{aligned}
$$

From (1.2) and (2.7), it is obvious that $U_{j}(s)$ converges uniformly and absolutely in the half-plane $\operatorname{Re}(s) \geq \frac{1}{2}+\varepsilon$ for any $\varepsilon>0$. This completes the proof of Lemma 2.1 .

Based on the work of Cogdell and Michel [1, Lau and Wu 11] showed that for $j=2,3,4, L\left(\operatorname{sym}^{j} f \times \operatorname{sym}^{j} f, s\right)$ has a meromorphic continuation to the whole complex plane and satisfies a functional equation.

Lemma 2.2. Let $f(z) \in S_{k}(\Gamma)$ be a Hecke eigenform of even integral weight $k$. The Rankin-Selberg L-function associated to $\mathrm{sym}^{j} f$ and $\mathrm{sym}^{j} f$ is defined as (2.6). For $j=2,3,4$, the Archimedean local factor of $L\left(\operatorname{sym}^{j} f \times \operatorname{sym}^{j} f, s\right)$ is

$$
L_{\infty}\left(\operatorname{sym}^{j} f \times \operatorname{sym}^{j} f, s\right)=\Gamma_{\mathbb{R}}(s)^{\delta_{2 \mid j}} \Gamma_{\mathbb{C}}(s)^{[j / 2]+\delta_{2 \nmid j}} \prod_{v=1}^{j} \Gamma_{\mathbb{C}}(s+v(k-1))^{j-v+1},
$$

where $\Gamma_{\mathbb{R}}(s)=\pi^{-s / 2} \Gamma(s / 2), \Gamma_{\mathbb{C}}(s)=2(2 \pi)^{-s} \Gamma(s), \delta_{2 \mid j}=1-\delta_{2 \nmid j}$, and

$$
\delta_{2 \nmid j}= \begin{cases}1, & \text { if } 2 \nmid j, \\ 0, & \text { otherwise } .\end{cases}
$$

Then the complete L-function

$$
\Lambda\left(\operatorname{sym}^{j} f \times \operatorname{sym}^{j} f, s\right)=: L_{\infty}\left(\operatorname{sym}^{j} f \times \operatorname{sym}^{j} f, s\right) L\left(\operatorname{sym}^{j} f \times \operatorname{sym}^{j} f, s\right)
$$

is entire except possibly for simple poles at $s=0,1$ and satisfies the functional equation

$$
\Lambda\left(\operatorname{sym}^{j} f \times \operatorname{sym}^{j} f, s\right)=\epsilon_{\mathrm{sym}^{j}} f \times \operatorname{sym}^{j} f \Lambda\left(\operatorname{sym}^{j} f \times \operatorname{sym}^{j} f, 1-s\right)
$$

with $\left|\epsilon_{\mathrm{sym}^{j} f \times \mathrm{sym}^{j} f}\right|=1$.

Proof. This is Proposition 2.1 in Lau and Wu [11]. 
Lemma 2.3. Let $j=2,3,4$. Then for any $\varepsilon>0$ and $0 \leq \sigma \leq 1$, we have

$$
L\left(\operatorname{sym}^{j} f \times \operatorname{sym}^{j} f, \sigma+i t\right) \ll_{f, \varepsilon}(1+|t|)^{\frac{(j+1)^{2}}{2}(1-\sigma)+\varepsilon} .
$$

Proof. From Lemma 2.2, we can follow standard arguments to establish the convexity bound for $L\left(\operatorname{sym}^{j} f \times \operatorname{sym}^{j} f, \sigma+i t\right)$ in the critical strip $\frac{1}{2} \leq \sigma \leq 1$ (see, for example, Chapter 5 of [7]).

Lemma 2.4. Let $j=2,3,4$. Then for $T \geq T_{0}$ (where $T_{0}$ is sufficiently large), we have the estimate

$$
\int_{T}^{2 T}\left|L\left(\operatorname{sym}^{j} f \times \operatorname{sym}^{j} f, \frac{1}{2}+i t\right)\right|^{2} d t \ll_{f, \varepsilon} T^{\frac{(j+1)^{2}}{2}+\varepsilon},
$$

where $\varepsilon$ is any positive constant.

Proof. From Lemma 2.2, we observe that the $L$-function $L\left(\operatorname{sym}^{j} f \times \operatorname{sym}^{j} f, s\right)$ is of degree $(j+1)^{2}$ and is being extended as an entire function except possibly with simple poles at $s=0$ and $s=1$. It also satisfies a nice functional equation of the Riemann zeta type, and thus we can write the functional equation here as

$$
L\left(\operatorname{sym}^{j} f \times \operatorname{sym}^{j} f, s\right)=\chi(s) L\left(\operatorname{sym}^{j} f \times \operatorname{sym}^{j} f, 1-s\right),
$$

where

$$
|\chi(s)| \asymp|t|^{\frac{(j+1)^{2}}{2}(1-2 \sigma)} \quad \text { as } \quad|t| \rightarrow \infty
$$

in any fixed strip $a \leq \sigma \leq b$. Now we follow the arguments of (i) of Theorem 4.1 of the paper by Sankaranarayanan [15]. The only necessary changes are that we need the free parameters $Y$ and $Y_{1}$ therein to be $Y=Y_{1}=c T^{\frac{(j+1)^{2}}{2}}$, where $c$ is a suitable positive constant. This leads to the estimate of this lemma.

\section{Proof of Theorems 1.1.-1.3}

Recall that for $j=2,3,4$, we define

$$
L_{j}(s)=\sum_{n=1}^{\infty} \frac{\lambda^{2}\left(n^{j}\right)}{n^{s}}
$$

for $\operatorname{Re}(s)>1$. From Lemma 2.1 and Lemma 2.2, we learn that $L_{j}(s)=L\left(\operatorname{sym}^{j} f \times\right.$ $\left.\operatorname{sym}^{j} f, s\right) U_{j}(s)$ can be analytically continued to the half-plane $\operatorname{Re}(s)>1 / 2$. In this region, $L_{j}(s)$ only has a simple pole $s=1$.

Now we begin to prove our main results. By (3.1) and Perron's formula (see Proposition 5.54 in [7]), we have

$$
\sum_{n \leq x} \lambda^{2}\left(n^{j}\right)=\frac{1}{2 \pi i} \int_{b-i T}^{b+i T} L_{j}(s) \frac{x^{s}}{s} d s+O\left(\frac{x^{1+\varepsilon}}{T}\right),
$$

where $b=1+\varepsilon$ and $1 \leq T \leq x$ is a parameter to be chosen later. Here we have used (1.2). 
Next we move the integration to the parallel segment with $\operatorname{Re}(s)=\frac{1}{2}+\varepsilon$. By Cauchy's residue theorem, we have

$$
\begin{aligned}
\sum_{n \leq x} \lambda^{2}\left(n^{j}\right)= & \operatorname{Res}_{s=1} L_{j}(s) x+\frac{1}{2 \pi i}\left\{\int_{\frac{1}{2}+\varepsilon-i T}^{\frac{1}{2}+\varepsilon+i T}+\int_{\frac{1}{2}+\varepsilon+i T}^{b+i T}+\int_{b-i T}^{\frac{1}{2}+\varepsilon-i T}\right\} L_{j}(s) \frac{x^{s}}{s} d s \\
& +O\left(\frac{x^{1+\varepsilon}}{T}\right) \\
= & : c_{j-1} x+I_{1}+I_{2}+I_{3}+O\left(\frac{x^{1+\varepsilon}}{T}\right) .
\end{aligned}
$$

For $I_{1}$, by Lemma 2.1, we have

$$
\begin{aligned}
& I_{1} \ll x^{\frac{1}{2}+\varepsilon}+x^{\frac{1}{2}+\varepsilon} \int_{1}^{T}\left|L\left(\operatorname{sym}^{j} f \times \operatorname{sym}^{j} f, 1 / 2+\varepsilon+i t\right) U_{j}(1 / 2+\varepsilon+i t)\right| t^{-1} d t \\
& (3.4) \ll x^{\frac{1}{2}+\varepsilon}+x^{\frac{1}{2}+\varepsilon} \int_{1}^{T}\left|L\left(\operatorname{sym}^{j} f \times \operatorname{sym}^{j} f, 1 / 2+\varepsilon+i t\right)\right| t^{-1} d t .
\end{aligned}
$$

Then by the Cauchy-Schwarz inequality, Gabriel convexity and Lemma 2.4, we have

$$
\begin{gathered}
I_{1} \ll x^{\frac{1}{2}+\varepsilon}+x^{\frac{1}{2}+\varepsilon} \log T \max _{T_{1} \leq T}\left\{\frac{1}{T_{1}} \int_{T_{1} / 2}^{T_{1}}\left|L\left(\operatorname{sym}^{j} f \times \operatorname{sym}^{j} f, 1 / 2+\varepsilon+i t\right)\right| d t\right\} \\
\times x^{\frac{1}{2}+\varepsilon} \log T \max _{T_{1} \leq T}\left\{\frac{1}{T_{1}}\left(\int_{T_{1} / 2}^{T_{1}}\left|L\left(\operatorname{sym}^{j} f \times \operatorname{sym}^{j} f, 1 / 2+\varepsilon+i t\right)\right|^{2} d t\right)^{\frac{1}{2}}\right. \\
\left.\times\left(\int_{T_{1} / 2}^{T_{1}} 1 d t\right)^{\frac{1}{2}}\right\}+x^{\frac{1}{2}+\varepsilon} \\
\ll x^{\frac{1}{2}+\varepsilon}+x^{\frac{1}{2}+\varepsilon} T^{\frac{(j+1)^{2}}{4}-\frac{1}{2}+\varepsilon} \ll x^{\frac{1}{2}+\varepsilon} T^{\frac{(j+1)^{2}}{4}-\frac{1}{2}+\varepsilon} .
\end{gathered}
$$

For the integrals over the horizontal segments, we use Lemma 2.3 to get

$$
\begin{aligned}
I_{2}+I_{3} & \ll \int_{\frac{1}{2}+\varepsilon}^{b} x^{\sigma}\left|L\left(\operatorname{sym}^{j} f \times \operatorname{sym}^{j} f, \sigma+i T\right)\right| T^{-1} d \sigma \\
(3.6) & \ll \max _{\frac{1}{2}+\varepsilon \leq \sigma \leq b} x^{\sigma} T^{\frac{(j+1)^{2}}{2}(1-\sigma)+\varepsilon} T^{-1}=\max _{\frac{1}{2}+\varepsilon \leq \sigma \leq b}\left(\frac{x}{T^{\frac{(j+1)^{2}}{2}}}\right)^{\sigma} T^{\frac{(j+1)^{2}}{2}-1+\varepsilon} \\
& \ll \frac{x^{1+\varepsilon}}{T}+x^{\frac{1}{2}+\varepsilon} T^{\frac{(j+1)^{2}}{4}-1+\varepsilon} .
\end{aligned}
$$

From (3.3), (3.5) and (3.6), we have

$$
\sum_{n \leq x} \lambda^{2}\left(n^{j}\right)=c_{j-1} x+O\left(\frac{x^{1+\varepsilon}}{T}\right)+O\left(x^{\frac{1}{2}+\varepsilon} T^{\frac{(j+1)^{2}}{4}-\frac{1}{2}+\varepsilon}\right) .
$$

On taking $T=x^{\frac{2}{(j+1)^{2}+2}}$ in (3.7), we have

$$
\sum_{n \leq x} \lambda^{2}\left(n^{j}\right)=c_{j-1} x+O\left(x^{1-\frac{2}{(j+1)^{2}+2}+\varepsilon}\right) .
$$


By taking $j=2,3,4$ respectively, we have

$$
\begin{aligned}
& \sum_{n \leq x} \lambda^{2}\left(n^{2}\right)=c_{1} x+O\left(x^{\frac{9}{11}+\varepsilon}\right), \\
& \sum_{n \leq x} \lambda^{2}\left(n^{3}\right)=c_{2} x+O\left(x^{\frac{8}{9}+\varepsilon}\right),
\end{aligned}
$$

and

$$
\sum_{n \leq x} \lambda^{2}\left(n^{4}\right)=c_{3} x+O\left(x^{\frac{25}{27}+\varepsilon}\right) .
$$

\section{ACKNOWLEDGEMENTS}

The first author is thankful to Professor Xiumin Ren, Professor Jianya Liu, and Professor Wenguang Zhai for their encouragement. The second author wishes to thank the Institute for Number Theory and Probability Theory, University of Ulm, Germany, for its generous support.

\section{REFERENCES}

[1] J. Cogdell and P. Michel, On the complex moments of symmetric power $L$-functions at $s=1$, Int. Math. Res. Not., 31(2004), 1561-1617. MR2035301(2005f:11094)

[2] P. Deligne, La Conjecture de Weil, I, Inst. Hautes Études Sci. Publ. Math., 43(1974), 273-307. MR0340258 (49:5013)

[3] O. M. Fomenko, On the behavior of automorphic $L$-functions at the center of the critical strip, Zap. Nauchn. Sem. S.-Peterburg. Otdel. Mat. Inst. Steklov. (POMl), 276(2001), Anal. Teor. Chisel i Teor. Funkts., 17, 300-311, 354; translation in J. Math. Sci. (N. Y.), 118(2003), 4910-4917. MR 1850374 (2002g:11060)

[4] S. Gelbart and H. Jacquet, A relation between automorphic representations of GL(2) and GL(3), Ann. Sci. École Norm. Sup., 11(1978), 471-542. MR.533066 (81e:10025)

[5] A. Ivić, On sums of Fourier coefficients of cusp forms, IV International Conference "Modern Problems of Number Theory and Its Applications": Current Problems, Part II (Russian) (Tula, 2001), 92-97, Mosk. Gos. Univ. im. Lomonosova, Mekh.-Mat. Fak., Moscow, 2002. MR:1985942 (2004d:11030)

[6] H. Iwaniec, Topics in classical automorphic forms, Graduate Studies in Math., vol. 17, Amer. Math. Soc., Providence, RI, 1997. MR/1474964 (98e:11051)

[7] H. Iwaniec and E. Kowalski, Analytic Number Theory, Amer. Math. Soc. Colloquium Publ. 53, Amer. Math. Soc., Providence, RI, 2004. MR2061214 (2005h:11005)

[8] H. Kim, Functoriality for the exterior square of $\mathrm{GL}_{4}$ and the symmetric fourth of $\mathrm{GL}_{2}$, Appendix 1 by D. Ramakrishnan, Appendix 2 by H. Kim and P. Sarnak, J. Amer. Math. Soc., 16(2003), 139-183. MR1937203 (2003k:11083)

[9] H. Kim and F. Shahidi, Functorial products for $\mathrm{GL}_{2} \times \mathrm{GL}_{3}$ and the symmetric cube for $\mathrm{GL}_{2}$ (with an appendix by C. J. Bushnell and G. Henniart), Ann. of Math. (2), 155(2002), 837-893. MR1923967 (2003m:11075)

[10] H. Kim and F. Shahidi, Cuspidality of symmetric powers with applications, Duke Math. J., 112(2002), 177-197. MR.1890650(2003a:11057)

[11] Y.-K. Lau and J. Wu, A density theorem on automorphic $L$-functions and some applications, Trans. Amer. Math. Soc., 358(2006), 441-472. MR2171241 (2006g:11097)

[12] G. S. Lü, On sums of Fourier coefficients of cusp forms over sparse sequences, to appear in Science in China, Ser. A.

[13] R. A. Rankin, Contributions to the theory of Ramanujan's function $\tau(n)$ and similar arithmetical functions, II. The order of the Fourier coefficients of the integral modular forms, Proc. Cambridge Phil. Soc., 35(1939), 357-372.

[14] R. A. Rankin, Sums of cusp form coefficients. Automorphic forms and analytic number theory (Montreal, PQ, 1989), 115-121, Univ. Montreal, Montreal, QC, 1990. MR1111014|(92g:11042) 
[15] A. Sankaranarayanan, Fundamental properties of symmetric square $L$-functions, I, Illinois J. Math., 46(2002), 23-43. MR.1936073 (2004a:11038)

[16] A. Sankaranarayanan, On a sum involving Fourier coefficients of cusp forms, Lithuanian Mathematical Journal, 46(2006), 459-474. MR2320364 (2008c:11068)

[17] A. Selberg, Bemerkungen über eine Dirichletsche Reihe, die mit der Theorie der Modulformen nahe verbunden ist, Arch. Math. Naturvid., 43(1940), 47-50. MR0002626 (2:88a)

[18] F. Shahidi, Third symmetric power $L$-functions for GL(2), Compos. Math., 70(1989), 245-273. MR.1002045 (90m:11081)

Department of Mathematics, Shandong Normal University, Jinan Shandong, 250014, People's Republic of China

E-mail address: laohuixue@sina.com

School of Mathematics, Tata Institute of Fundamental Research, Homi Bhabha Road, Mumbai-400 005, India - And - Institute for Number Theory and Probability Theory, University of Ulm, D-89069, Ulm, Germany

E-mail address: sank@math.tifr.res.in

E-mail address: ayyadurai.sankaranarayanan@uni-ulm.de 